\title{
STABILIZATION OF RESISTIVE KINK MODES IN THE TOKAMAK
}

BY

A. H. GLASSER, $H, P$, FURTH, AND $P, H$, RUTHERFORD

\section{PLASMA PHYSICS LABORATORY}

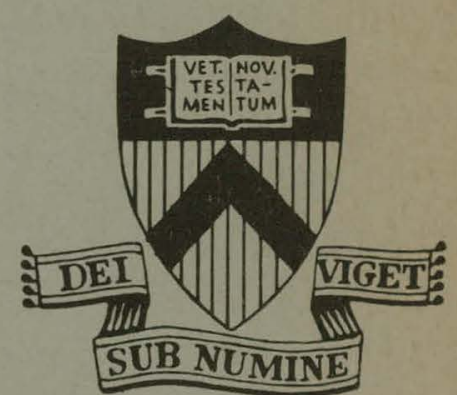

DISTRIBUTION OF THIS DOCUM**T IS UNLIMITED

\section{PRINCETON UNIVERSITY PRINCETON, NEW JERSEY}

This work was supported by U. S. Energy Research and Development Administration Contract E(11-1)-3073. Reproduction, translation, publication, use and disposal, in whole or in part, by or for the United States Government is permitted. 


\section{DISCLAIMER}

This report was prepared as an account of work sponsored by an agency of the United States Government. Neither the United States Government nor any agency Thereof, nor any of their employees, makes any warranty, express or implied, or assumes any legal liability or responsibility for the accuracy, completeness, or usefulness of any information, apparatus, product, or process disclosed, or represents that its use would not infringe privately owned rights. Reference herein to any specific commercial product, process, or service by trade name, trademark, manufacturer, or otherwise does not necessarily constitute or imply its endorsement, recommendation, or favoring by the United States Government or any agency thereof. The views and opinions of authors expressed herein do not necessarily state or reflect those of the United States Government or any agency thereof. 


\section{DISCLAIMER}

Portions of this document may be illegible in electronic image products. Images are produced from the best available original document. 


\section{NOTICE}

This report was prepared as an account of work sponsored by the United States Government. Neither the United States nor the United States Energy Research and Development Administration, nor any of their employees, nor any of their contractors, subcontractors, or their employees, makes any warranty, express or implied, or assumes any legal liability or responsibility for the accuracy, completeness or usefulness of any information, apparatus, product or process disclosed, or represents that its use would not infringe privately owned rights.

Printed in the United States of America.

Available from

National Technical Information Service

U, S. Department of Commerce 5285 Port Royal Road

Springfield, Virginia 22151

Price: Printed Copy \$_; Microfiche $\$ 1.45$

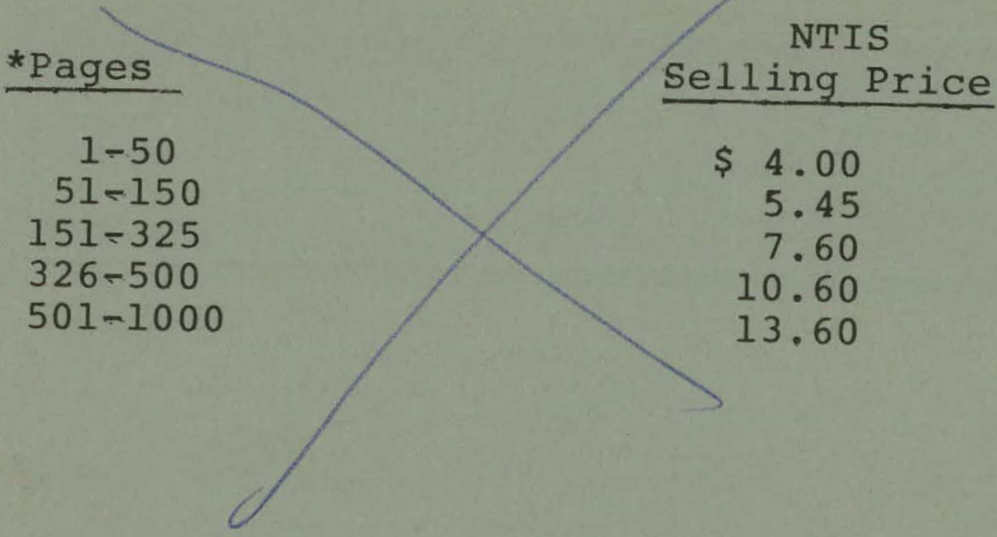


Stabilization of Resistive Kink Modes in the Tokamak ${ }^{\dagger}$

A. H. Glasser, H. P. Furth, and P. H. Rutherford

Plasma Physics Laboratory, Princeton University

Princeton, New Jersey 08540

\begin{abstract}
Optimized current profiles are shown to be capable of providing simultaneous stability against all resistive kink modes in the tokamak.
\end{abstract}

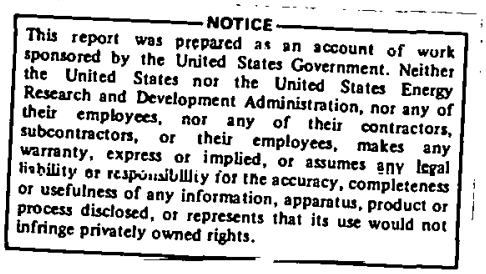


The utility of the tokamak increases with the ratio $\mathrm{B}_{\theta} / \mathrm{B}_{\phi}$ of poloidal to toroidal field strength. In order to avoid unstable helical MHD perturbations (kink modes) of the form exp $i(m \theta-n \phi)$, the "safety factor" $q(r) \equiv 2 \pi / \iota(r)=$ $\mathrm{rB}_{\phi} / \mathrm{RB}_{\theta}$, must, however, be restricted. ${ }^{l}$ If there is a radial range wherein $q(r)<1$, then the fundamental mode $m=1$, $\mathrm{n}=1$ is unstable, ${ }^{1}$ whether or not the plasma is perfectly conducting at the point where $q(r)=1$. Higher modes $(m>1)$ can be unstable only as resistive kink, or tearing, modes $^{2}$ for which the singular point, where $q(\mathrm{~s})=\mathrm{m} / \mathrm{n}$, falls into a resistive region.

The object of this letter is to demonstrate the existence of q-profiles that provide simultaneous stability against all the low-m kink modes, while minimizing the limiter value $q_{a}=q(a)$. The principle is contained in a comparison theorem ${ }^{2}$ that states: for two profiles of the rotational transform $\iota$ having the same shear $(d \iota / d r)_{s}$ and the same transform, $\iota_{s}$ at the singular point of a given mode, if the two profiles everywhere satisfy $\left|\iota_{1}(r)-\iota_{s}\right|>\left|\iota_{2}(r)-\iota_{s}\right|$, then $\iota_{1}(r)$ is llore stable against the given mode than $\iota_{2}(r)$. Resistive kink instabilities can also be eliminated (in sufficiently hot plasmas) by a local pressure gradient at $s$ due to favorable average toroidal curvature, ${ }^{3}$ or by the proximity of a perfectly conducting exterior shell.

To illustrate optimum profiles, we will consider two cases: Case $A$, a profile with $q_{a}>2$, giving stability against ail finite-m modes, without need of a conducting 
shell or of toroidal-curvature effects; and Case B, a similar profile, but adding a conducting shell to achieve stability at $q_{a}<2$

We consider first a straight cylindrical configuration, and neglect pressure-gradient effects. The magnetic perturbations outside the resistive layer satisfy the equation for a marginal MHD mode, namely ${ }^{1,2}$

$$
\frac{1}{r^{2}} \cdot \frac{\partial}{\partial r}\left(r^{2} \frac{\partial \psi}{\partial r}\right)-\frac{m^{2}}{r^{2}} \psi=\frac{m}{r F} \frac{d j}{d r} \psi
$$

where $\psi=\operatorname{irB}_{r} / \mathrm{m}$ is the perturbed poloidal flux function, $j=d\left(r B_{\theta}\right) / r d r$ is the equilibrium longitudinal current density, and

$$
F=\vec{k} \cdot \vec{B}=(m-n q) B_{\theta} / r
$$

For a marginal resistive mode, Eq. (1) is satisfied everywhere, i.e., there is no discontinuity at the singular surface.

Analytic solutions of $\mathrm{Eq}$. (1) can readily be found for the model of $j(r)$ shown in Fig. 1. In this model, there is a central current channel of radius $r=c$ with uniform current density $j(r)=j_{1}$, surrounding by a "pedestal" of radius $r=a$ with uniform, but lower, current density $j(r)=j_{2}$. For $r>a$, the current density vanishes, so that the limiter could be placed just outside $r=a$, with $a$ conducting shell at $r=b$. 
Within $0<r<c$, the solution of Eq.(1) is given by

$$
\psi / \psi_{\mathrm{C}}=(r / \mathrm{c})^{\mathrm{m}}
$$

Across $r=c$, the matching conditions are that $\psi=\psi_{c}$ be continuous, and that

$$
\frac{\left[\psi^{\prime}\right]_{c}}{\psi_{c}}=\frac{m}{c F_{c}}[j]_{c}=-\frac{2 m(1 . . p)}{c\left(m-n q_{c}\right)}
$$

where []$_{C}$ denotes the discontinuity across $r=c$, and $p=j_{2} / j_{1}$, is a factor describing the height of the pedestal. (Given $q_{c}$ and $q_{a}$, values of $p$ are possible within the range $0<\dot{p}<\ddot{q}_{c} / q_{a} ;$ the relation

$$
\frac{c^{2}}{a^{2}}=\frac{q_{e^{/ q}}-p}{1-p}
$$

then determines the ratio c/a.) Applying these matching conditions at $r=c$, the solution of Eq. (1) within $c<r<a$ is given by

$$
\frac{\psi}{\psi_{c}}=\left(\frac{1}{r}\right)^{m}-\frac{1-\mu}{m-n q_{c}}\left[\left(\frac{1}{r}\right)^{m}-\left(\frac{\varepsilon}{r}\right)^{m}\right]
$$

Across $r=a$, the matching conditions are again that $\psi=\psi_{a}$ be continuous and that 


$$
\frac{\left[\psi^{\prime}\right]_{a}}{\psi_{a}}=\frac{m}{a F_{a}}[j]_{a}=-\frac{2 m q_{a} p}{a q_{c}\left(m-n q_{a}\right)}
$$

Applying these matching conditions at $r=a$, and using Eq. (6) at $r=a$ for $\psi_{a^{\prime}}$ the solution of Eq. (1) for $r>a$ is given by

$$
\begin{gathered}
\frac{\psi}{\psi_{c}}=\left(\frac{r}{c}\right)^{m}-\frac{1-p}{m-n q_{c}}\left[\left(\frac{r}{c}\right)^{m}-\left(\frac{c}{r}\right)^{m}\right] \\
-\frac{q_{a} p}{q_{c}\left(m-n q_{a}\right)}\left[\left(\frac{r}{a}\right)^{m}-\left(\frac{a}{r}\right)^{m}\right]\left\{\left(\frac{a}{c}\right)^{m}-\frac{1-p}{m-n q_{c}}\left[\left(\frac{a}{c}\right)^{m}-\left(\frac{c}{a}\right)^{m}\right]\right\}^{m}
\end{gathered}
$$

If the conducting shell is absent $(b \rightarrow \infty)$, the stability condition (i.e., the condition that no marginal mode exists) is that the coefficient of the $r^{m}$. term be positive, i.e.,

$$
1-\frac{1-p}{m-n q_{c}}>\frac{q_{a} p}{q_{c}\left(m-n q_{a}\right)}\left\{1-\frac{1-p}{m-n q_{c}}\left[1-\left(\frac{c}{a}\right)^{2 m}\right]\right\}
$$

Suppose, first, that the pedestal is entirely absent, i.e., $p=0$. In this case, instability occurs if $0<m-\mathrm{nq}_{\mathrm{C}}$ < 1. For the $(m, n)=(2,1)$ mode to be stable, it is clearly necessary to have $q_{C}<1$, in which case the $(m, n)=(1,1)$ mode is unstable. Morcover, if $q_{C}$ is just above 1 , the entire sequence of modes $(2,1),(3,2),(4,3)$, etc., is unstable. Even if the $\mathrm{m}=2$ mode were stabilized by means of a fairly close conducting shell, the higher-m modes of this sequence would, 
typically, remain unstable, since the effect of the shell falls off rapidly with rising $m$. With the $m=2$ mode stabilized by a conducting shell, one might consider setting $q_{C}$ just above 1.5, so that the modes $(3,2),(4,3)$, etc., become stable. However, in: this case, the sequence of modes $(5,3),(8,5)$, etc., would be: unstable. It is, thus, of considerable interest to determine whether, in either case, a current profile with a nonzero pedestal can provide simultaneous stability against all. modes.

Let us consider case $A$, in which $q_{C}$ is just above 1 , and $q_{a}$ is just above 2 , with a finite value of $p$ in the range $0<p<0.5$. If $q_{C}$ is infinitesimally above 1 , Eq. (9) shows clearly that the "inner" sequence of modes $(2,1),(3,2)$, $(4,3)$, etc., whose singular surfaces fall into the pedestal region, is. positively stable, since in each case the left side of Eq. (9) is positive and the right side is negative. We must also, however, demonstrate the stability of the "outer" sequence of modes $(3,1),(5,2),(7,3)$, etc., whose singular surfaces fall outside the pedestal region. A condition stronger than (9) wnuld rcsult from replacing $(\mathrm{c} / \mathrm{a})^{2 \mathrm{~m}}$, by $(\mathrm{c} / \mathrm{a})^{2}$; we do this, and substitute Eq. (5) for $\mathrm{c} / \mathrm{a}$, to obtain the sufficient stability condition $(1-2 p)\left(m-n q_{c}-1\right)>0$, after using $m-n q_{a}=1$ and $q_{a} / q_{c}=2$. Since $p<q_{c} / q_{a}=0.5$, this condition is always satisfied by the modes of the "outer" sequence, which have $m-n q_{C} 22$.

If $q_{c}$ and $q_{a}$ exceed 1 and 2 , respectively, by small 
but finite increments, a reasonable number of the modes in both the "inner" and "outer" sequences can be made positively stable. This is illustrated in Fig. 2, for the case where $q_{C}=1.05$ and $\mathrm{q}_{\mathrm{a}}=2.1$, and for various values of the pedestal $\mathrm{p}$. We see that, in this case, the optimum value for $p$, in the sense of stabilizing the greatest range of low m-values $(m<8)$, is about 0.3 .

Let us now consider Case $B$, which requires a conducting shell to stabilize the $m=2$ mode, but offers the advantage that the limiter $q$-value can be dropped below 2. Here $q_{C}$ is again just above 1 , but $\mathrm{q}_{a}$ is chosen to be just above 1.5, with a value of: $p$ in the range $0<p<2 / 3$. As before, if $q_{C}$ is infinitesimally above 1, Eq. (9) shows that the "inner" sequence of modess with $(m, n)=(3,2),(4,3)$, etc., is positively stable, for any finite value of $\mathrm{p}$ in the above range. We must also, however, demonstrate the stability of the "outer" sequence of modes $(2,1),(5,3),(8,5)$, etc. For $q_{a}$ infinitesimally above 1.5, we find that the stability condition is never satisfied for the $(2,1)$ mode, but it can be satisfied for the $(5,3)$ mode, and all higher modes of this "outer". sequence, provided $p<0.32$. The $(2,1)$ mode can, however, be stabilized by means of a conducting shell. The requirement on the radius $b$ of the shell can be determined trom Eq. (8) by setting $\psi_{\mathrm{C}}>0$ at $\mathrm{r}^{\mathrm{2}}=\mathrm{b}$. Employing $q_{c}=1, q_{a}=1.5, E q .(5)$ for $c / a$, and $(m, n)=(2,1)$, we obtain

$$
b / a<(4 / 3 p)^{1 / 4}
$$


For $p \simeq 0.3$, this gives $b / a<1.45$ a requirement that could be met rather easily.

: The simple analytic treatment given above has the advantage of clarifying the role of a current pedestal in stabilizing low-m modes. However, as we have seen, the use of a discontinuous function for $j(r)$ has the disadvantage of exciting high-m modes. $:$ In order to investigate the possiblity of stabilizing all kink modes simultaneously, we have employed a computer program that determines the stabjlity of arhitrary smonth curront profiles by calculating the quantities $\Delta^{\prime}$ that measure the potential energy perturbations for the various modes. Figures $3 \mathrm{~A}$ and $3 \mathrm{~B}$ show two examples of "realistic" current profiles resembling the analytic cases $A$ and $B$. In both cases, we see that the entire spectrum of modes is stabilized $\left(\Delta^{\prime}<0\right)$, the higher-m modes apparently being suppressed by the smoothing of $j(r)$. The corresponding limiter q-values are 2.6 and 1.8 , respectively. In Fig. 3B a fairly close conducting shell was needed $(b / a=1.2)$; alternatively, one could invoke toroidal-curvature stabilization ${ }^{3}$ of the weakly unstable higherm modes of the "inner" sequence, thus permitting a lower pedestal and a larger value of $\mathrm{b} / \mathrm{a}$.

our results are in accord with the experimentally observed destabilizing effects of limiter q-values that approach 2 , or high levels of impurity influx. In either case, the outer plasma region would be cooled, so that the pedestal on the current profile would tend to be truncated short of the $q(r)=2$ 
point. In larger tokamaks, it may be possible to achieve better control over the current distribution, so that profiles resembling Fig. 3A could be approximated.

Experiments on conducting-shell stabilization ${ }^{4}$ proved successful in suppressing the $(m, n)=(2,1)$ mode, thus obtaining gross stability at $q_{a}<2$. There was, however, evidence of a deterioration in confinement, particularly for $q_{a} \simeq 1.5$. our results for case $B$ show that this could be explained in terms of the truncation of the pedestal on the current profile short of the point where $q(r)=1.5$. 


\section{REFERENCES}

†This work was supported byiU. S. Energy Research and Development Administration Contract E(11-1)-3073.

1V. D. Shafranov, Zh. Tekh. Fiz. 40, 241 (1970)

[Sov. Phys. - Tech. Phys. 15, 175 (1970)].

2.il. F. Furth, F. Il. Rútieifuld, dad H. Selbery, Phys. Fluids 16, 1054 (1973):

${ }^{3}$ A. H. Glasser, J. M. Greene, and J. L. Johnson, Phys. Fluids 19, 567 (1976).

${ }^{4}$ v. S. Vlasenkov, V. M. Leonov, V. G. Merezhkin, and V. S. Mukhovatov, Third International Symposium on Toroidal Plasma Confinement, Max-Planck-Institute for Plasma Physics, Garching, Germany (1973). 


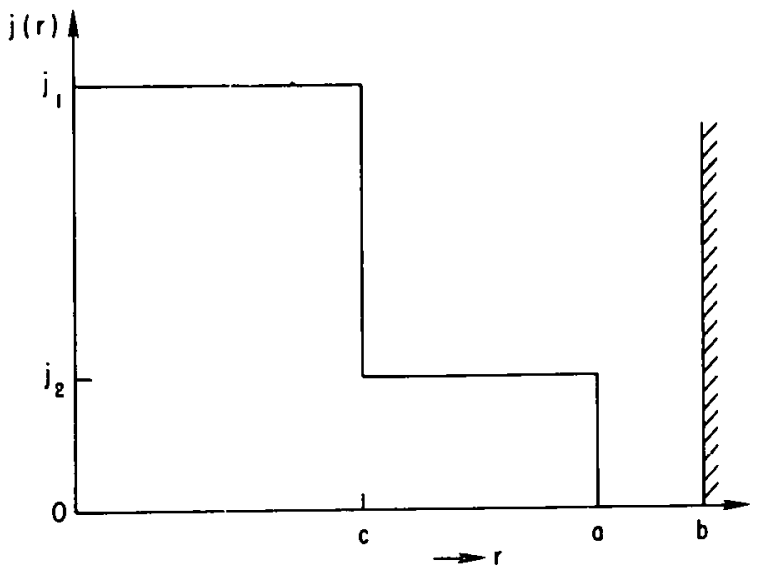

762250

Fig. 1. Simple model of $j(r)$ used in the analytic calculation.

762249

Fig. 2. Stability diagram for modes $(m, n)$ in Case $A$ using the current profile of Fig. 1 , with $q_{c}=1.05, q_{a}=2.1, b=\infty$, and varjous values of the pedestal $p$.

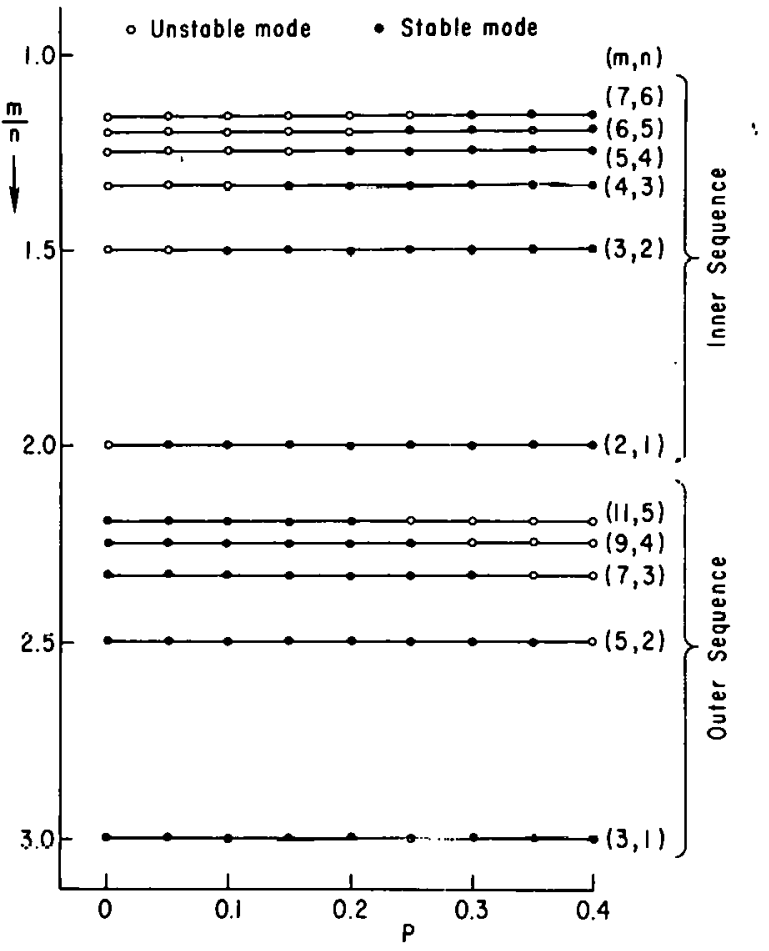




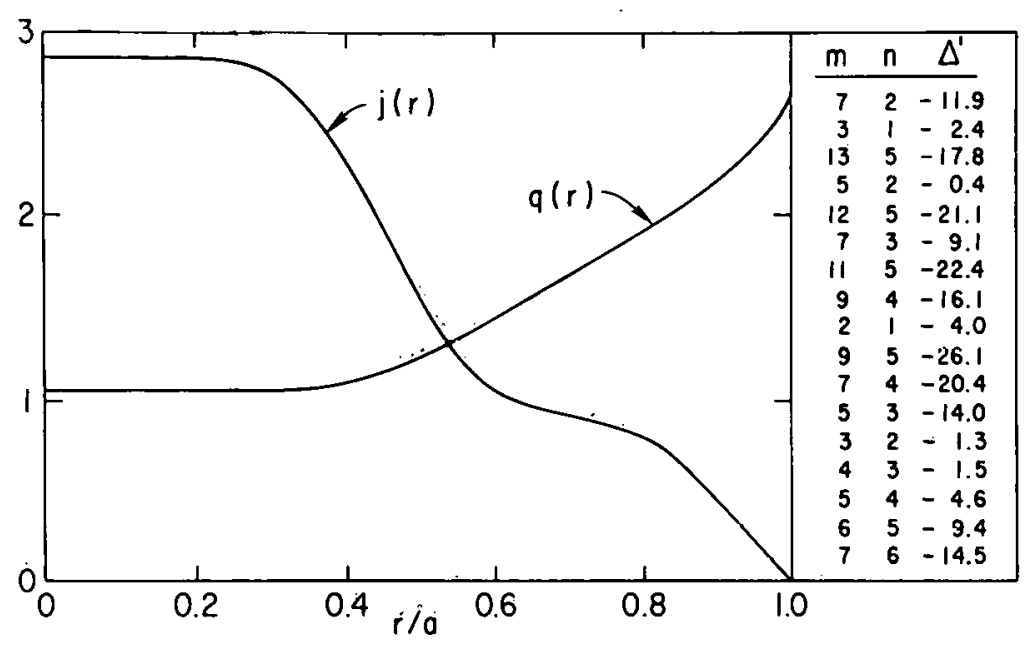

762252

Fig. 3(a). Example of a stable profile similar to Case $A$, with no conducting shell; the computed values of $\Delta^{\prime}$ show that all the indicated modes are stable.

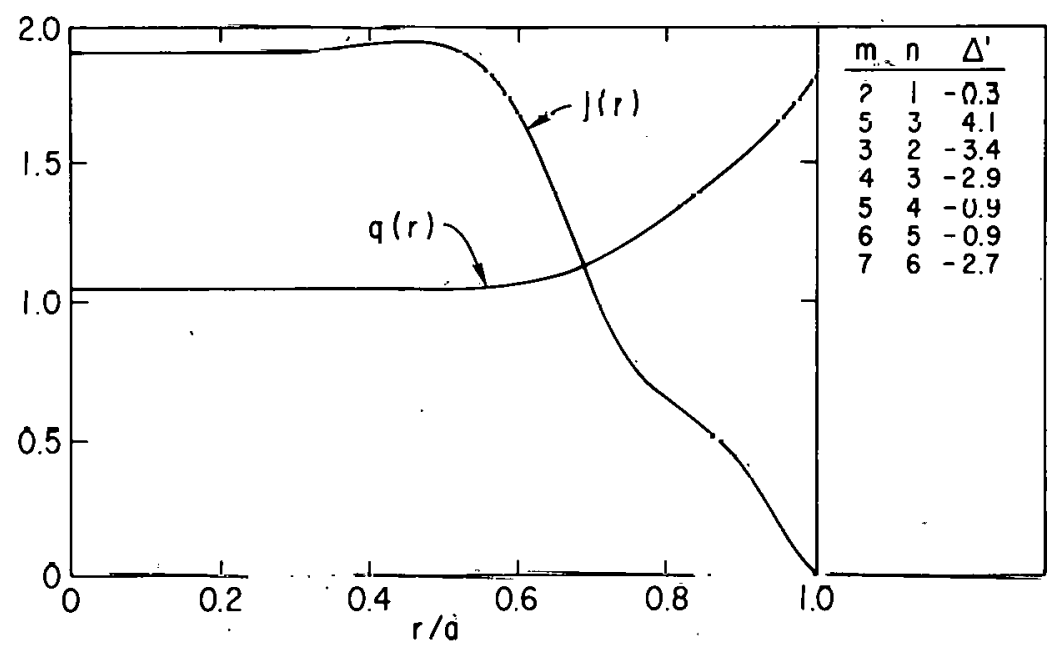

762251

Fig. 3(b). Example of a stable profile similar to Case $B$, with a conducting shell at $r / a=1.2$. 